\title{
MiR-195-3p is a Novel Prognostic Biomarker Associated with Immune Infiltrates of Lung Adenocarcinoma
}

\author{
Yi Lao' \\ Taidong $\mathrm{Li}^{2}$ \\ Xin Xie ${ }^{1}$ \\ Kangbiao Chen' \\ Ming $\mathrm{Li}^{\prime}$ \\ Lu Huang iD ' \\ 'The Fourth Department of Medical \\ Oncology, Central Hospital of \\ Guangdong Nongken, Zhanjiang Cancer \\ Hospital, Zhanjiang, Guangdong, 524002, \\ People's Republic of China; ${ }^{2}$ Department \\ of Thoracic Surgery, Central Hospital of \\ Guangdong Nongken, Zhanjiang Cancer \\ Hospital, Zhanjiang, Guangdong, 524002, \\ People's Republic of China
}

Background: MicroRNA-195-3p (miR-195-3p) plays an important role in some tumors, but its role in LUAD is unclear. This study explored the expression of miR-195-3p in LUAD and the relationship between the expression of miR-195-3p and the clinical and prognostic characteristics of LUAD patients.

Methods: MiR-195-3p expression and clinical information of LUAD patients were obtained from The Cancer Genome Atlas (TCGA). Kruskal-Wallis test, Wilcoxon signed rank test, logistic regression, and Cox regression were used to assess the relationship between the expression level of miR-195-3p and clinical features in LUAD tissues. Kaplan-Meier survival curves were used to analyze the effect of miR-195-3p expression levels on the prognosis of LUAD patients. Target genes of miR-195-3p were predicted by several software. GO (Gene Ontology), KEGG (Kyoto Encyclopedia of Genes and Genomes), and immune infiltration analysis were used to analyze the possible regulatory network of miR-195-3p.

Results: Compared with normal lung tissue, miR-195-3p is down expressed in LUAD tissue $(\mathrm{P}<0.001)$. The low miR-195-3p expression in LUAD was significantly associated with $\mathrm{N}$ stage $(\mathrm{P}=0.046)$, pathologic stage $(\mathrm{P}=0.011)$, and gender $(\mathrm{P}=0.010)$. Low miR-195-3p expression predicted a poorer overall survival (HR: $0.60 ; 95 \% \mathrm{CI}: 0.45-0.81 ; \mathrm{P}=0.001$ ) and disease-specific survival (HR: 0.55 ; $95 \% \mathrm{CI}: 0.37-0.80 ; \mathrm{P}=0.002$ ). The expression of miR195-3p (HR: 0.488 ; 95\% CI: 0.304-0.784; $\mathrm{P}=0.003$ ) was independently correlated with OS in LUAD patients. High expression of miR-195-3p genes, including ABCC2, AGMAT, ARNTL2, ATP6V0A4, CDC25A, CDK1, FAM111B, GJB2, GRIP1, HMGA2, HOXA9, KIF14, SYT2, and TFAP2A, were associated with poor OS in LUAD. GO and KEGG analysis suggested that miR-195-3p was related to the phagosome pathway. MiR-195-3p may promote the function of B cells, dendritic cells, eosinophils, immature dendritic cells, macrophages, Mast cells, NK cells, plasmacytoid dendritic cells, and follicular helper T cells. Conclusion: Low miR-195-3p expression is significantly associated with poor survival in LUAD, which may be a promising prognostic biomarker for LUAD.

Keywords: lung adenocarcinoma, miR-195-3p, gene expression, prognosis, immune infiltration, biomarker

\section{Introduction}

Lung cancer is one of the malignant tumors that seriously threaten human health. ${ }^{1}$ Lung cancer accounts for $11.6 \%$ of all cancers and has the highest incidence, with 18.4 deaths from each type of cancer. $^{2}$ Non-small cell lung cancer (NSCLC) accounts for $80 \%$ of all lung cancers and is the most common histological form of lung cancer. ${ }^{3}$ Lung adenocarcinoma (LUAD) is the most aggressive histological
The Fourth Department of Medical Oncology, Central Hospital of Guangdong Nongken, Zhanjiang Cancer Hospital, No. 2 Mid Renmin Avenue, Zhanjiang, 524002, Guangdong, People's Republic of China

Tel +86-0759-2633696

Email luternh@I63.com 
type of lung cancer, and the incidence of LUAD has been increasing rapidly. ${ }^{4}$ Compared with other types of lung cancer, LUAD has a slower growth rate and has no specific early clinical symptoms. ${ }^{5}$ Due to the similarity of symptoms of common respiratory diseases, it is easy to misdiagnose. ${ }^{6}$ Approximately $75 \%$ of patients are diagnosed with advanced or metastatic cancer and often miss major surgery opportunities. With the improvement of modern clinical diagnosis methods, although the overall survival rate and quality of life have improved, the survival rate is still unsatisfactory, only about $15 \%{ }^{7}$ Therefore, there is an urgent need to find validated tumor markers for LUAD to guide clinical diagnosis.

MicroRNAs (miRNAs or miRs), typically 18-23 nucleotides in length, have received widespread attention as oncogenes or inhibitors that regulate gene activity at the post-transcriptional and translational levels. ${ }^{8}$ Numerous studies have shown that aberrantly expressed miRNAs in various types of cancer are associated with cell development, cell proliferation, apoptosis, and tumorigenesis. ${ }^{9,10}$ miR-195-3p was a clinical biomarker for renal cell carcinoma. ${ }^{11}$ Cancer is a very heterogeneous disease with complex interactions between malignant cells and the tumor microenvironment (TME). ${ }^{12}$ The TME is composed of various immune cells, mesenchymal-derived cells, and extracellular matrix (ECM), which influence all stages of tumorigenesis by interacting directly with tumor cells. ${ }^{13}$ MiR-195 inhibits NSCLC by targeting CHEK1. ${ }^{14}$ MiR-195 suppresses lung adenocarcinogenesis by regulating $\mathrm{CD} 4+\mathrm{T}$ cell activation through the CCDC88C/Wnt signaling pathway. ${ }^{15}$ Hsa-miR-195-3p acts as one of the maturators of hsa-miR-195. However, the expression of miR-195-3p in LUAD and its relationship with clinical features and immune infiltration are still unclear.

The Cancer Genome Atlas (TCGA) is a landmark cancer genomics program that has molecularly characterized more than 20,000 primary cancers and matched normal samples across 33 cancer types. TCGA has generated more than 2.5 PB of genomic, epigenomic, transcriptomic, and proteomic data. These data have improved our ability to diagnose, treat, and prevent cancer and continue to be publicly available to anyone in the research community. Based on the Cancer Genome Atlas (TCGA) database, this study analyzed the expression of miR-195-3p in LUAD and normal lung tissues, the relationship between miR-195-3p expression and the clinical characteristics of patients, and the relationship between 195-3p expression and prognosis in LUAD patients. Target genes of miR-195-
$3 p$ were predicted by several software. Enrichment analysis, and immune infiltration analysis were used to analyze the possible regulatory network of miR-195$3 p$. The results of this study may provide a promising prognostic biomarker for LUAD.

\section{Materials and Methods}

\section{Clinical Information}

Analytical methods referred to the published reference. ${ }^{16}$ Molecule: hsa-miR-195-3p [MIMAT0004615]. The data includes miRNAseq data and clinical data from the BCGSC level 3 miRNA profile analysis of the TCGA (https://portal. gdc.cancer.gov/) and LUAD (lung adenocarcinoma) project.

\section{Differential Expression of miR-195-3p in LUAD Patients}

\section{Unpaired Samples}

Analytical methods referred to the published reference. ${ }^{17}$ Molecule: hsa-miR-195-3p. Data transformation: $\log 2$ transformation of miRNAseq data in RPM format for inter-sample expression comparison. Data Filtering: Retain paired samples.

\section{Paired Samples}

Analytical methods referred to the published reference. ${ }^{17}$ Molecule: hsa-miR-195-3p. Data transformation: $\log 2$ transformation of miRNAseq data in RPM format for inter-sample expression comparison. Data Filtering: None.

\section{ROC Curves}

Analytical methods referred to the published reference. ${ }^{16}$ The molecule is hsa-miR-195-3p. Clinical variables: Normal vs Tumor. Disease: LUAD.

\section{The Correlation Between miR-195-3p Expression and Clinical Information in LUAD Patients} Clinical Relevance

Analytical methods referred to the published reference. ${ }^{16}$ The molecule is hsa-miR-195-3p. The subgroup is median. The clinical variables include $\mathrm{N}$ stage, pathological stage, and gender.

\section{Logistics Analysis}

Analytical methods referred to the published reference. ${ }^{16}$ Dependent variable: hsa-miR-195-3p. 


\section{The Relationship Between the Expression of miR-195-3p and the Prognosis of LUAD Patients \\ Kaplan-Meier Method}

Analytical methods referred to the published reference. ${ }^{16}$ Molecule: hsa-miR-195-3p. Prognosis type: overall survival (OS) and disease specific survival (DSS).

\section{Cox Regression}

Analytical methods referred to the published references. ${ }^{17,18}$ Prognosis type: Overall Survival. Included variables: clinical characteristics and hsa-miR-195-3p.

\section{Target Gene Prediction of miR-195-3p}

The target genes of miR-195-3p were predicted by several databases. ${ }^{19-22}$ The LUAD mRNA average expression file was downloaded from the website (http://bioinfo.life.hust. edu.cn/miR path/download.html). Venn diagram was used to obtain the shared genes of miR-195-3p target genes and LUAD down-regulated genes.

\section{The Correlation Between Expression of miR-195-3p Target Genes and Prognosis}

Analytical methods referred to the published reference. ${ }^{23}$ Molecules: ABCC2 [ENSG00000023839], AGMAT [ENSG00000116771], ARNTL2 [ENSG00000029153], CDC25A [ENSG00000164045], CDK1 [ENSG0000017 0312], FAM111B [ENSG00000189057], GJB2 [ENSG000 00165474], GRIP1 [ENSG00000155974], HMGA2 [ENS G00000149948], HOXA9 [ENSG00000078399], KIF14 [ENSG00000118193], TFAP2A [ENSG00000137203]. Grouping: $0-50$ vs 50-100. Prognosis type: overall survival (OS). Data: RNAseq data and clinical data from level 3 HTSeq-FPKM format from the TCGA LUAD project.

\section{Gene Ontology (GO) and Kyoto Encyclopedia of Genes and Genomes (KEGG) Analysis}

The DAVID database ${ }^{24,25}$ was utilized to perform GO and KEGG analysis for miR-195-3p targets.

\section{Sing Sample Gene Set Enrichment Analysis (SsGSEA) Analysis of Immune Infiltration}

Analytical methods referred to the published references. ${ }^{13,23,26}$ Molecule: hsa-miR-195-3p.

\section{Analysis of Statistics}

Analytical methods referred to the published reference. ${ }^{23}$

\section{Results}

\section{Clinical Characteristics}

There were 521 patients in this study (Table 1). According to the T stage, we had 173 patients with T1 (33.4\%), 277 patients with T2 (53.5\%), 49 patients with T3 (9.5\%), and 19 patients with T4 (3.7\%). The N stage included $336 \mathrm{~N} 0$ (66.3\%), $95 \mathrm{~N} 1$ (18.7\%), $74 \mathrm{~N} 2$ (14.6\%), and $2 \mathrm{~N} 3$ (0.4\%). The M stage included 351 N0 (93.9\%), and 23 N1 (6.1\%). The pathological stage included 283 Stage I (55.1\%), 123 Stage II (23.9\%), 84 Stage III (16.3\%), and 24 Stage IV (4.7\%). According to the primary therapy outcome, we had 68 patients with PD (15.7\%), 35 patients with SD (8.1\%), 6 patients with PR (1.4\%), and 324 patients with CR (74.8\%). According to the gender, we had 278 females (53.4\%) and 243 males (46.6\%). According to race, we had 8 Asian patients (1.8\%), 53 Black or African American patients (11.6\%), and 394 White patients $(86.6 \%)$. According to age, there were 240 patients $(\leq 65$, $47.8 \%)$ and 262 patients $(>65,52.2 \%)$. According to the residual tumor, there were $342 \mathrm{R} 0$ patients (95.3\%), $13 \mathrm{R} 1$ patients (3.6\%), and $4 \mathrm{R} 2$ patients (1.1\%). According to the anatomic neoplasm subdivision, there were 202 patients with left $(39.9 \%)$ and 304 patients with right (60.1\%). According to the anatomic neoplasm subdivision 2, there were 62 central lungs (33.3\%) and 124 peripheral lungs $(66.7 \%)$. The number pack years smoked included 176 patients $(<40,49.7 \%)$ and 178 patients $(\geq 40,50.3 \%)$. The smokers included 76 no (15\%) and 431 yes (85\%). The median age was 66.5 years, with a range of 59 to 72 years.

\section{Correlation Between miR-195-3p Expression and Clinical Features in LUAD Patients}

MiR-195-3p expression in LUAD tissues was significantly lower than that in normal lung tissues $(1.990 \pm 0.030, \mathrm{n}=46$ vs $2.629 \pm 0.157, \mathrm{n}=521, \mathrm{P}<0.001$ ) (Figure $1 \mathrm{~A}$ ). The expression of MiR-195-3p in in LUAD tissues (1.734 $\pm 0.084, \mathrm{n}=46$ ) was significantly lower than that of matched normal tissues $\quad(2.629 \pm 0.157, \quad \mathrm{n}=46) \quad(\mathrm{P}<0.001)$ (Figure 1B). The AUC of miR-195-3p was 0.659 (Figure 1C). MiR-195-3p expression was related to gender $(\mathrm{P}=0.012)$ (Table 1). The low expression of miR-195-3p in LUAD patients is significantly associated with $\mathrm{N}$ stage 
Table I Clinical Information of LUAD Patients and Correlation Between miR-195-3p Expression and Clinical Features in LUAD Patients (TCGA)

\begin{tabular}{|c|c|c|c|c|c|}
\hline Characteristic & Overall & $\begin{array}{l}\text { Low Expression of } \\
\text { hsa-miR-1 } 95-3 p\end{array}$ & $\begin{array}{l}\text { High Expression of } \\
\text { hsa-miR-195-3p }\end{array}$ & $\mathbf{p}$ & Method \\
\hline $\mathrm{n}$ & & 260 & 261 & & \\
\hline T stage, $n(\%)$ & & & & 0.057 & Chisq. test \\
\hline TI & 173 (33.4\%) & 74 (14.3\%) & $99(19.1 \%)$ & & \\
\hline $\mathrm{T} 2$ & $277(53.5 \%)$ & I 48 (28.6\%) & $129(24.9 \%)$ & & \\
\hline T3 & 49 (9.5\%) & $24(4.6 \%)$ & $25(4.8 \%)$ & & \\
\hline T4 & $19(3.7 \%)$ & $13(2.5 \%)$ & $6(1.2 \%)$ & & \\
\hline $\mathrm{N}$ stage, $\mathrm{n}(\%)$ & & & & 0.074 & Fisher test \\
\hline No & $336(66.3 \%)$ & $161(31.8 \%)$ & 175 (34.5\%) & & \\
\hline $\mathrm{NI}$ & 95 (18.7\%) & $50(9.9 \%)$ & 45 (8.9\%) & & \\
\hline N2 & $74(14.6 \%)$ & $47(9.3 \%)$ & $27(5.3 \%)$ & & \\
\hline N3 & $2(0.4 \%)$ & I (0.2\%) & I $(0.2 \%)$ & & \\
\hline M stage, $n(\%)$ & & & & 0.623 & Chisq. test \\
\hline Mo & 351 (93.9\%) & 187 (50\%) & 164 (43.9\%) & & \\
\hline MI & $23(6.1 \%)$ & $14(3.7 \%)$ & $9(2.4 \%)$ & & \\
\hline Pathologic stage, n (\%) & & & & 0.088 & Chisq. test \\
\hline Stage I & $283(55.1 \%)$ & $134(26.1 \%)$ & $149(29 \%)$ & & \\
\hline Stage II & $123(23.9 \%)$ & $58(11.3 \%)$ & $65(12.6 \%)$ & & \\
\hline Stage III & $84(16.3 \%)$ & $51(9.9 \%)$ & $33(6.4 \%)$ & & \\
\hline Stage IV & 24 (4.7\%) & $15(2.9 \%)$ & $9(1.8 \%)$ & & \\
\hline Primary therapy outcome, $n$ (\%) & & & & 0.060 & Fisher test \\
\hline PD & $68(15.7 \%)$ & $40(9.2 \%)$ & $28(6.5 \%)$ & & \\
\hline SD & $35(8.1 \%)$ & $12(2.8 \%)$ & $23(5.3 \%)$ & & \\
\hline PR & $6(1.4 \%)$ & $4(0.9 \%)$ & $2(0.5 \%)$ & & \\
\hline CR & $324(74.8 \%)$ & 147 (33.9\%) & 177 (40.9\%) & & \\
\hline Gender, n (\%) & & & & 0.012 & Chisq. test \\
\hline Female & $278(53.4 \%)$ & 124 (23.8\%) & 154 (29.6\%) & & \\
\hline Male & $243(46.6 \%)$ & $136(26.1 \%)$ & 107 (20.5\%) & & \\
\hline Race, n (\%) & & & & 0.538 & Fisher test \\
\hline Asian & $8(1.8 \%)$ & $4(0.9 \%)$ & $4(0.9 \%)$ & & \\
\hline Black or African American & $53(11.6 \%)$ & $22(4.8 \%)$ & $31(6.8 \%)$ & & \\
\hline White & $394(86.6 \%)$ & 195 (42.9\%) & 199 (43.7\%) & & \\
\hline Age, n (\%) & & & & 0.861 & Chisq. test \\
\hline$\leq 65$ & $240(47.8 \%)$ & $|2|(24.1 \%)$ & $119(23.7 \%)$ & & \\
\hline$>65$ & $262(52.2 \%)$ & 129 (25.7\%) & I33 (26.5\%) & & \\
\hline Residual tumor, n (\%) & & & & 0.917 & Fisher test \\
\hline Ro & $342(95.3 \%)$ & $183(5 \mid \%)$ & 159 (44.3\%) & & \\
\hline RI & $13(3.6 \%)$ & $8(2.2 \%)$ & $5(1.4 \%)$ & & \\
\hline R2 & $4(1.1 \%)$ & $2(0.6 \%)$ & $2(0.6 \%)$ & & \\
\hline Anatomic neoplasm subdivision, $\mathrm{n}(\%)$ & & & & 0.703 & Chisq. test \\
\hline Left & $202(39.9 \%)$ & $104(20.6 \%)$ & 98 (19.4\%) & & \\
\hline Right & $304(60.1 \%)$ & $150(29.6 \%)$ & $154(30.4 \%)$ & & \\
\hline Anatomic neoplasm subdivision 2, n (\%) & & & & 0.638 & Chisq. test \\
\hline Central Lung & $62(33.3 \%)$ & 37 (19.9\%) & $25(13.4 \%)$ & & \\
\hline Peripheral Lung & $124(66.7 \%)$ & $68(36.6 \%)$ & $56(30.1 \%)$ & & \\
\hline number_pack_years_smoked, n (\%) & & & & 0.525 & Chisq. test \\
\hline$<40$ & $176(49.7 \%)$ & $87(24.6 \%)$ & $89(25.1 \%)$ & & \\
\hline$\geq 40$ & 178 (50.3\%) & $95(26.8 \%)$ & $83(23.4 \%)$ & & \\
\hline Smoker, n (\%) & & & & 0.070 & Chisq. test \\
\hline No & $76(15 \%)$ & $30(5.9 \%)$ & $46(9.1 \%)$ & & \\
\hline Yes & 431 (85\%) & $222(43.8 \%)$ & 209 (4I.2\%) & & \\
\hline Age, median (IQR) & & $66(58,72)$ & $67(59.75,74)$ & 0.111 & Wilcoxon \\
\hline
\end{tabular}



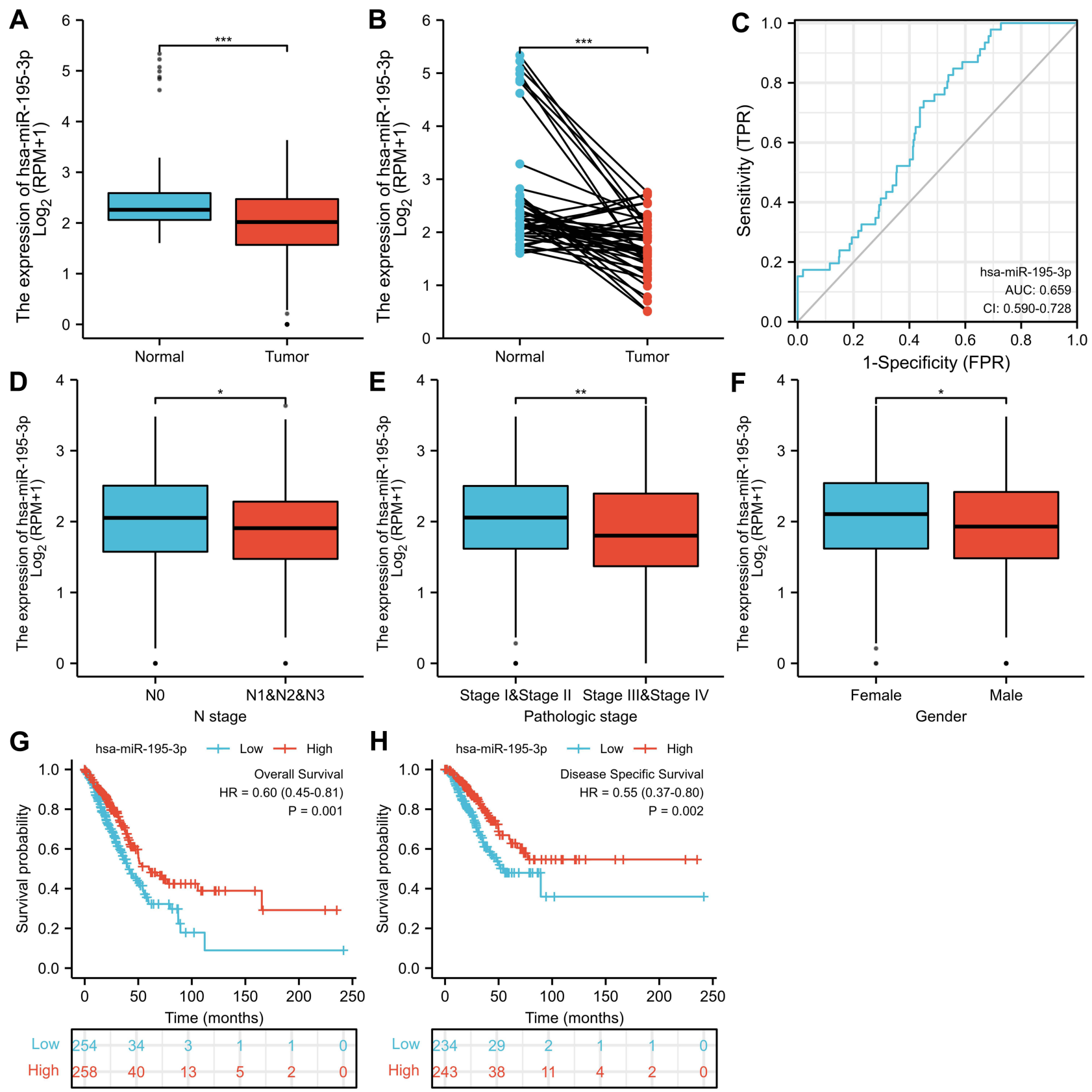

Figure I The expression and clinical significance of miR-195-3p in LUAD. (A) miR-195-3p expression was significantly lower in 52I LUAD tissues (I.990 \pm 0.030 ) compared with 46 normal tissues $(2.629 \pm 0.157)(P<0.001)$; (B) MiR-195-3p expression was significantly lower in 46 LUAD tissues $(1.734 \pm 0.084)$ compared with 46 normal tissues $(2.629 \pm 0.157)(P<0.05)$; (C) ROC curve; (D) N Stage; (E) pathologic stage; (F) gender; (G) overall survival, OS; (H) disease specific survival. Significance marker: * P<0.05; $* * \mathrm{P}<0.01$; *** $\mathrm{P}<0.001$.

$(\mathrm{P}=0.046)$, pathologic stage $(\mathrm{P}=0.011)$, and gender $(\mathrm{P}=0.010)$ (Figure 1D-F, and Table 2).

\section{Correlation Between miR-195-3p Expression and Prognosis in LUAD Patients}

Low expression of miR-195-3p in LUAD patients was associated with poor OS (HR: 0.60 ; 95\% CI: $0.45-0.81$;
$\mathrm{P}=0.001$ ) and DSS (HR: 0.55; 95\% CI: 0.37-0.80; $\mathrm{P}=0.002$ ) (Figure $1 \mathrm{G}-\mathrm{H}$ ). The univariate analysis of the Cox regression model of the OS prognostic factors in Table 3 showed that low miR-195-3p expression levels (HR: 0.592; 95\% CI: 0.440-0.796; $\mathrm{P}<0.001$ ) were related to T stage (HR: $2.385 ; 95 \% \mathrm{CI}: 1.634-3.481 ; \mathrm{P}<0.001$ ), $\mathrm{N}$ stage (HR: 2.617; 95\% CI:1.946-3.519; $\mathrm{P}<0.001$ ), $\mathrm{M}$ stage (HR: 2.330; 95\% CI: 1.360-3.991; $\mathrm{P}=0.002$ ), pathologic stage (HR: 2.766; 95\% CI: 2.030-3.768; 
Table 2 Expression of miR-195-3p in Relation to Clinical Characteristics (Logistic Regression)

\begin{tabular}{|l|l|l|l|}
\hline Characteristics & Total (N) & Odds Ratio (OR) & P value \\
\hline T stage (T3\&T4 vs TI\&T2) & 518 & $0.816(0.487-1.359)$ & 0.436 \\
N stage (NI\&N2\&N3 vs N0) & 507 & $0.685(0.472-0.992)$ & 0.046 \\
M stage (MI vs M0) & 374 & $0.733(0.298-1.716)$ & 0.481 \\
Primary therapy outcome (PD\&SD\&PR vs CR) & 433 & $0.786(0.508-1.214)$ & 0.278 \\
Pathologic stage (Stage III \& Stage IV vs Stage I \& Stage II) & 514 & $0.571(0.368-0.877)$ & 0.011 \\
Gender (Male vs Female) & 521 & $0.633(0.447-0.895)$ & 0.010 \\
Race (Black or African American \& White vs Asian) & 455 & $1.060(0.248-4.532)$ & 0.935 \\
Age (>65 vs S65) & 502 & $1.048(0.738-1.489)$ & 0.792 \\
Residual tumor (RI\&R2 vs R0) & 359 & $0.806(0.287-2.147)$ & 0.668 \\
Anatomic neoplasm subdivision (Right vs Left) & 506 & $1.090(0.763-1.556)$ & 0.637 \\
Anatomic neoplasm subdivision 2 (Peripheral Lung vs Central Lung) & 186 & $1.219(0.659-2.278)$ & 0.531 \\
number_pack_years_smoked ( $\geq 40$ vs <40) & 354 & $0.854(0.562-1.296)$ & 0.459 \\
Smoker (Yes vs No) & 507 & $0.614(0.370-1.005)$ & 0.055 \\
\hline
\end{tabular}

Table 3 Association of OS and Clinical Characteristics in LUAD (Cox Regression)

\begin{tabular}{|c|c|c|c|c|c|}
\hline \multirow[t]{2}{*}{ Characteristics } & \multirow{2}{*}{$\begin{array}{l}\text { Total } \\
\text { (N) }\end{array}$} & \multicolumn{2}{|c|}{ Univariate Analysis } & \multicolumn{2}{|c|}{ Multivariate Analysis } \\
\hline & & HR (95\% Cl) & $P$ value & HR (95\% Cl) & $P$ value \\
\hline T stage (TI\&T2 vs T3\&T4) & 509 & $2.385(\mathrm{I} .634-3.48 \mathrm{I})$ & $<0.001$ & $1.802(0.855-3.797)$ & 0.121 \\
\hline $\mathrm{N}$ stage (N0 vs NI\&N2\&N3) & 498 & $2.617(1.946-3.519)$ & $<0.001$ & $1.607(0.980-2.632)$ & 0.060 \\
\hline M stage (M0 vs $M I)$ & 365 & $2.330(1.360-3.991)$ & 0.002 & $1.204(0.443-3.273)$ & 0.716 \\
\hline Pathologic stage (Stage I \& Stage II vs Stage III \& Stage IV) & 505 & $2.766(2.030-3.768)$ & $<0.001$ & $1.595(0.783-3.250)$ & 0.198 \\
\hline Primary therapy outcome (PD\&SD\&PR vs CR) & 426 & $0.368(0.26 \mathrm{I}-0.519)$ & $<0.001$ & $0.270(0.163-0.446)$ & $<0.001$ \\
\hline Gender (Female vs Male) & 512 & $1.024(0.765-1.37 \mid)$ & 0.873 & & \\
\hline Race (Asian vs Black or African American \& White) & 455 & $2.604(0.364-18.609)$ & 0.340 & & \\
\hline Age $(\leq 65$ vs $>65)$ & 502 & $1.210(0.900-1.625)$ & 0.206 & & \\
\hline Residual tumor (R0 vs RI\&R2) & 350 & $4.044(2.256-7.250)$ & $<0.001$ & $3.902(1.510-10.084)$ & 0.005 \\
\hline Anatomic neoplasm subdivision (Left vs Right) & 498 & $1.015(0.751-1.372)$ & 0.923 & & \\
\hline Anatomic neoplasm subdivision 2 (Central Lung vs & 179 & $0.922(0.575-1.479)$ & 0.737 & & \\
\hline Peripheral Lung) & & & & & \\
\hline number_pack_years_smoked ( $<40$ vs $\geq 40$ ) & 348 & $1.049(0.732-1.504)$ & 0.793 & & \\
\hline Smoker (No vs Yes) & 498 & $0.917(0.607-1.384)$ & 0.679 & & \\
\hline hsa-miR-195-3p (Low vs High) & 512 & $0.592(0.440-0.796)$ & $<0.001$ & $0.488(0.304-0.784)$ & 0.003 \\
\hline
\end{tabular}

$\mathrm{P}<0.001$ ), primary therapy outcome (HR: $0.368 ; 95 \% \mathrm{CI}$ : $0.261-0.519 ; \mathrm{P}<0.001$ ), and residual tumor (HR: 4.044; 95\% CI: 2.256-7.250; $\mathrm{P}<0.001)$. The multivariate analysis showed that miR-195-3p (HR: $0.488 ; 95 \%$ CI: $0.304-$ $0.784 ; \mathrm{P}=0.003$ ), primary therapy outcome (HR: 0.270 ; 95\% CI: 0.163-0.446; $\mathrm{P}<0.001)$, and residual tumor (HR:3.902; 95\% CI: 1.510-10.084; $\mathrm{P}=0.005$ ) were independently related to OS (Table 3 ).

\section{Relationship Between miR-195-3p Target Genes and Survival of LUAD Patients}

There were 470 miR-195-3p target genes, 3066 LUAD up-regulated genes, and 51 common genes in Figure 2.
High ABCC2 expression was related to poor OS (HR: 1.46; 95\% CI: 1.09-1.95; $\mathrm{P}=0.01$ ) (Figure 3A). High AGMAT expression was related to poor OS (HR: 1.52; 95\% CI: 1.14-2.03; P=0.005) (Figure 3B). High ARNTL2 expression was related to poor OS (HR: 1.62 ; 95\% CI: 1.21-2.17; $\mathrm{P}=0.001$ ) (Figure 3C). High CDC25A expression was related to poor OS (HR: $1.44 ; 95 \%$ CI: $1.078-$ 1.92; $\mathrm{P}=0.014$ ) (Figure 3D). High CDK1 expression was related to poor OS (HR: 1.84; 95\% CI: 1.37-2.48; $\mathrm{P}<0.001$ ) (Figure 3E). High FAM111B expression was related to poor OS (HR: 1.66; 95\% CI: 1.24-2.22; $\mathrm{P}=0.001$ ) (Figure 3F). High GJB2 expression was related to poor OS (HR: 1.75; 95\% CI: 1.31-2.35; $\mathrm{P}<0.001$ ) 


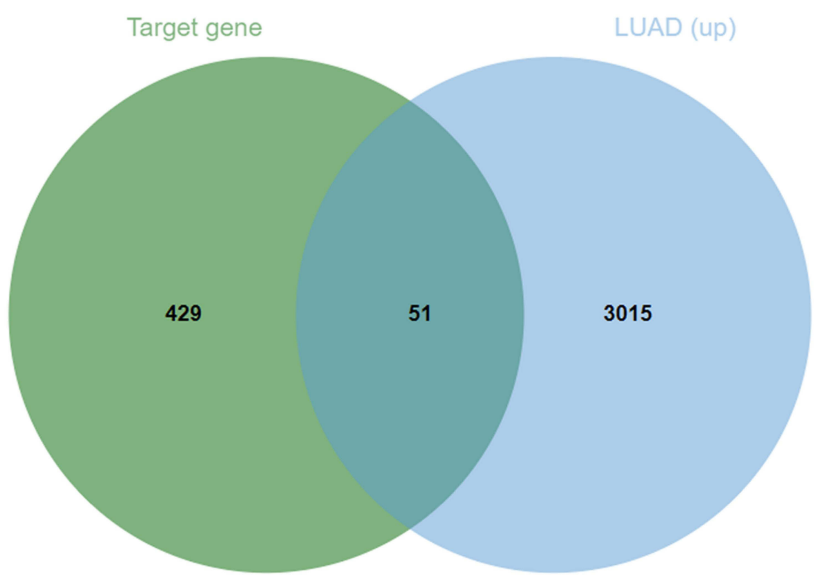

\begin{tabular}{|lllll|}
\hline ABCC2 & DCAF12L2 GALR1 & KIRREL2 & SYT2 \\
AGMAT & DCAF8L1 & GJB2 & LMNB1 & TAT \\
ARNTL2 & DGKB & GJB6 & LUZP4 & TFAP2A \\
ATP6V0A4 DMGDH & GRIP1 & MSTN & TFAP2B \\
BHMT & DUOX2 & HMGA2 & MUC7 & THBS2 \\
CDC25A & EN2 & HOXA10 & NNAT & TUBB2A \\
CDH12 & FAM111B & HOXA9 & NR1H4 & WNK3 \\
CDK1 & FAM133A & HOXD13 & PKHD1 & \\
CNTNAP2 FAP & ITGA2 & SLC26A4 & \\
COL10A1 & GABRA3 & KCNQ3 & SLC6A11 & \\
CPS1 & GABRA5 & KIF14 & SP8 & \\
\hline
\end{tabular}

Figure 2 The common genes between miR-195-3p target genes and LUAD upregulated genes. (Venn diagram).
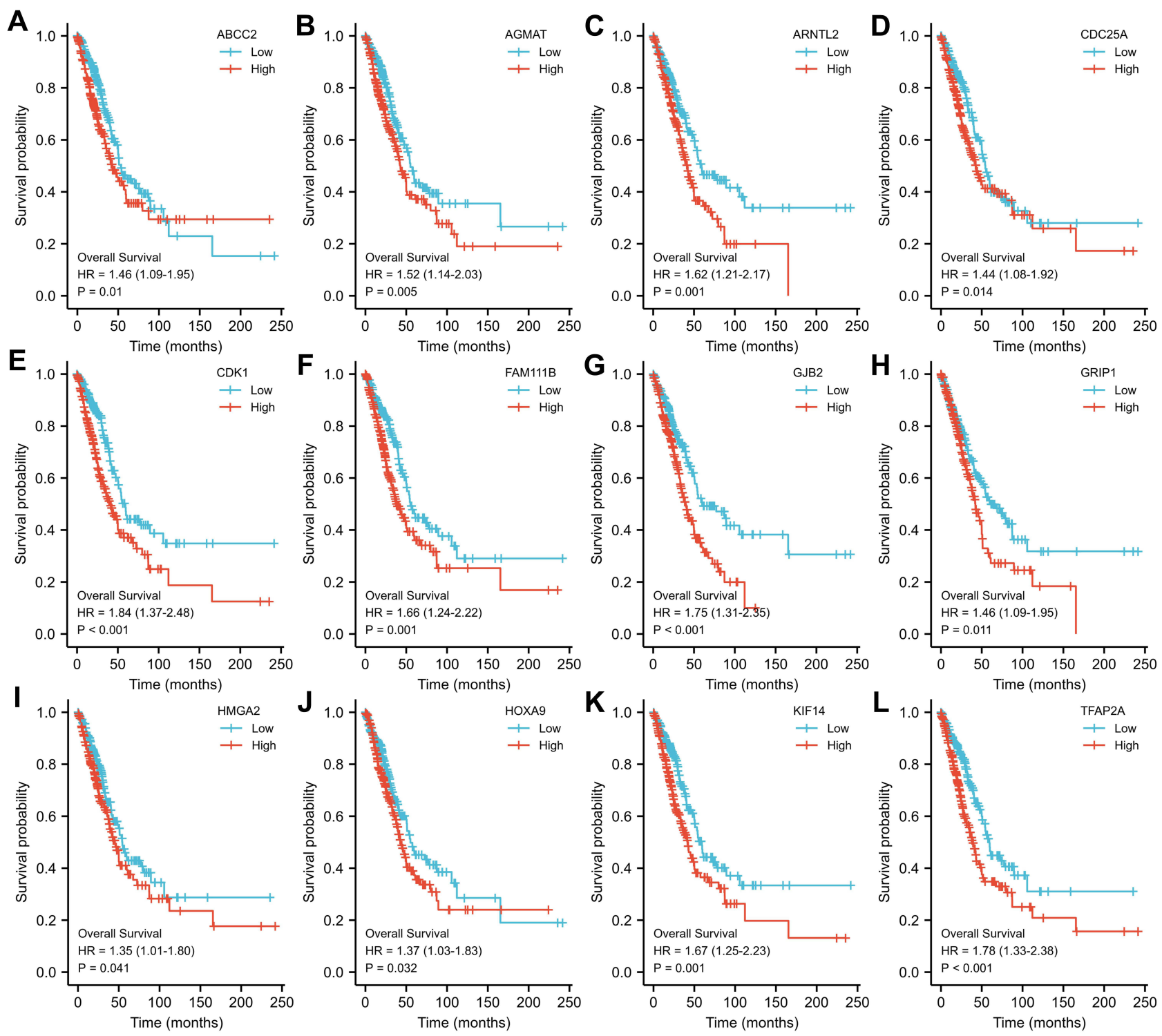

Figure 3 Correlation of miR-195-3p target gene expression with prognosis of LUAD patients. (A) ABCC2, (B) AGMAT, (C) ARNTL2, (D) CDC25CA, (E) CDKI, (F) FAMI I IB, (G) GJB2, (H) GRIPI, (I) HMGA2, (J) HOXA9, (K) KIFI4, and (L) TFAP2A. Overall survival, OS. 
(Figure 3G). High GRIP1 expression was related to poor OS (HR: 1.46; 95\% CI: 1.09-1.95; $\mathrm{P}=0.011$ ) (Figure 3H). High HMGA2 expression was related to poor OS (HR: 1.35; 95\% CI: 1.01-1.80; $\mathrm{P}=0.041$ ) (Figure 3I). High NHS expression was related to poor OS (HR: 1.37 ; $95 \% \mathrm{CI}$ : 1.03-1.83; $\mathrm{P}=0.032$ ) (Figure 3J). High NDN expression was related to poor OS (HR: 1.67; 95\% CI: $1.25-2.23$; $\mathrm{P}=0.001$ ) (Figure $3 \mathrm{~K}$ ). High NHS expression was related to poor OS (HR: $1.78 ; 95 \% \mathrm{CI}: 1.33-2.38 ; \mathrm{P}<0.001$ ) (Figure 3L).

\section{Possible Pathways Involved in MiR-195-3p Target Genes}

Common genes are involved in biological processes, including sensory perception of mechanical stimulus, sensory perception of sound, appendage morphogenesis, limb morphogenesis, and response to dexamethasone, etc.; cellular components including cell projection membrane, apical part of cell, cellcell junction, apical plasma membrane, and brush border membrane, etc.; molecular function including benzodiazepine receptor activity, GABA-gated chloride ion channel activity, inhibitory extracellular ligand-gated ion channel activity,
DNA-binding transcription activator activity, RNA polymerase II-specific, and GABA-A receptor activity, etc.; the phagosome pathway (Figure 4 and Table 4).

\section{The Correlation Between miR-195-3p Expression and Immune Infiltration}

The expression of miR-195-3p is positively correlated with the level of immune infiltration of $B$ cells $(0.311 \pm 0.077$ vs 0.286 $\pm 0.074, \mathrm{P}<0.001 ; \mathrm{r}=0.180$; Figures $5 \mathrm{~A}, 6 \mathrm{~A}$, and 7), dendritic cells (DC) $(0.489 \pm 0.099$ vs $0.45 \pm 0.109, \mathrm{P}<0.001 ; \mathrm{r}=0.240$; Figures $5 \mathrm{~B}, 6 \mathrm{~B}$, and 7$)$, Eosinophils $(0.399 \pm 0.028$ vs 0.391 $\pm 0.031, \mathrm{P}<0.001 ; \mathrm{r}=0.210$; Figures $5 \mathrm{C}, 6 \mathrm{C}$, and 7$)$, immature dendritic cells (iDC) $(0.534 \pm 0.059$ vs $0.506 \pm 0.06, \mathrm{P}<0.001$; $\mathrm{r}=0.280$; Figures $5 \mathrm{D}, 6 \mathrm{D}$, and 7$)$, Macrophages $(0.621 \pm 0.062$ vs $0.597 \pm 0.071, \mathrm{P}<0.001 ; \mathrm{r}=0.210$; Figures $5 \mathrm{E}, 6 \mathrm{E}$, and 7 ), Mast cells $(0.329 \pm 0.073$ vs $0.291 \pm 0.077, \mathrm{P}<0.001 ; \mathrm{r}=0.170$; Figures $5 \mathrm{~F}, 6 \mathrm{~F}$, and 7$)$, NK cells $(0.493 \pm 0.024$ vs $0.479 \pm 0.025$, $\mathrm{P}<0.001 ; \mathrm{r}=0.310$; Figures $5 \mathrm{G}, 6 \mathrm{G}$, and 7 ), plasmacytoid dendritic cells (pDC) $(0.613 \pm 0.102$ vs $0.570 \pm 0.098$, $\mathrm{P}<0.001 ; \mathrm{r}=0.290$; Figures $5 \mathrm{H}, 6 \mathrm{H}$ and 7 ), and follicular helper $\mathrm{T}$ cells (TFH) $(0.415 \pm 0.038$ vs $0.402 \pm 0.037, \mathrm{P}<0.001$; $\mathrm{r}=0.210$; Figures 5I, 6I and 7).

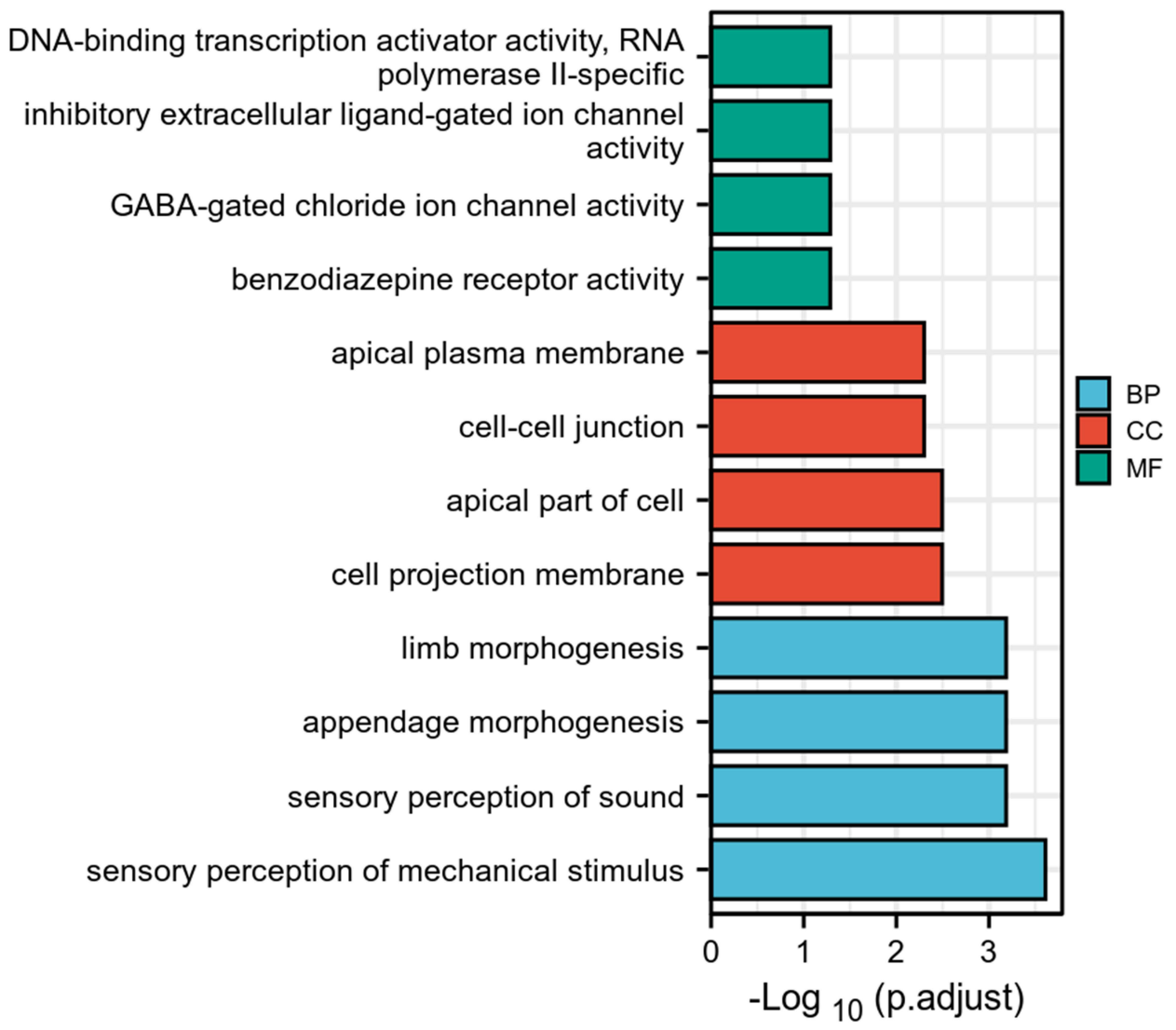

Figure $4 \mathrm{GO}$ analysis of common genes. 
Table 4 KEGG Analysis of Common Genes

\begin{tabular}{|l|l|l|l|l|l|}
\hline Category & Term & Count & $\%$ & P value & Genes \\
\hline KEGG_PATHWAY & hsa04I45: Phagosome & 4 & 7.84 & 0.013 & TUBB2A, ITGA2, ATP6V0A4, THBS2 \\
\hline
\end{tabular}

\section{Discussion}

MiRNAs are associated with the development of some cancers. miR-375 is critical to the advancement of LUAD. $^{27}$ MiR-139-5p may act as a tumor suppressor gene in the development and progression of NSCLC. ${ }^{28}$
Low expression of MiR-99a is a poor prognostic factor for patients with LUAD and acts as a tumor suppressor by targeting mTOR. ${ }^{29}$ miR-210-3p is a promising LUAD diagnostic biomarker. $^{30}$ Therefore, it is essential to develop new prognostic biomarkers for LUAD.
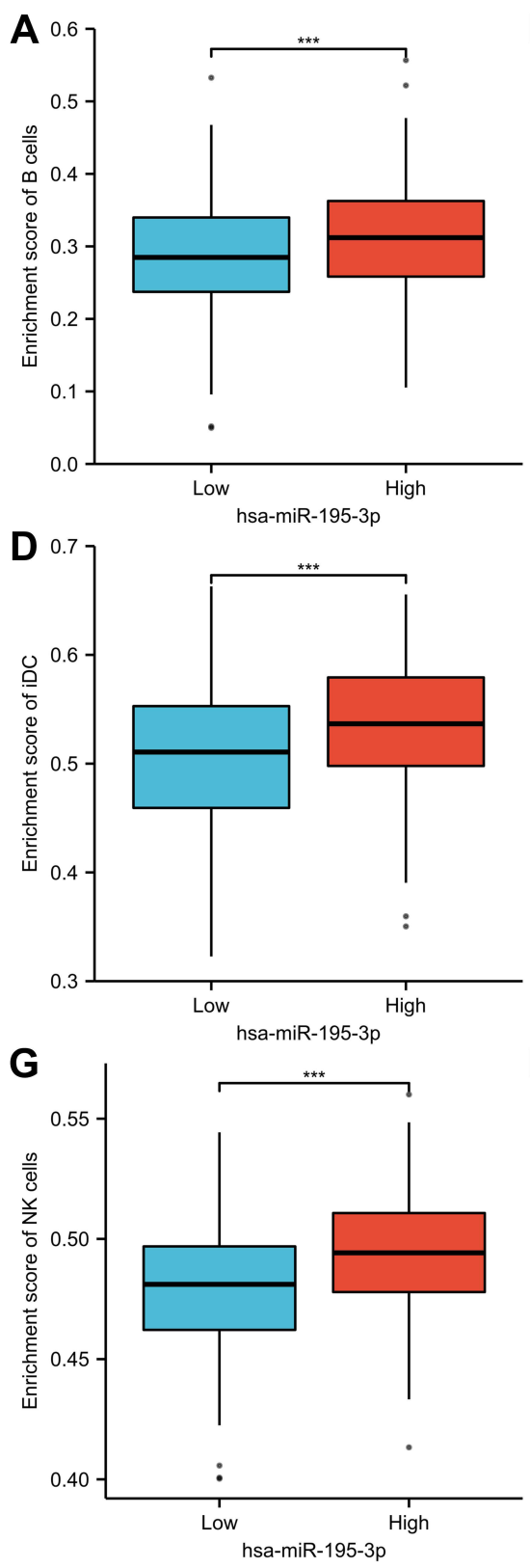
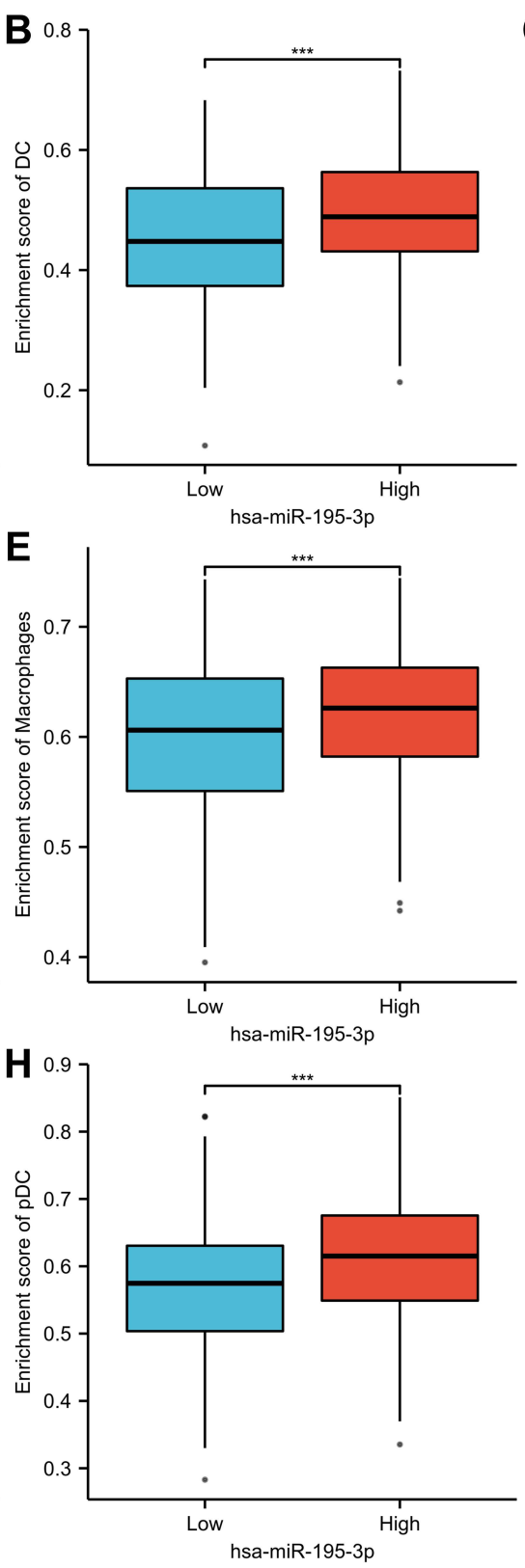
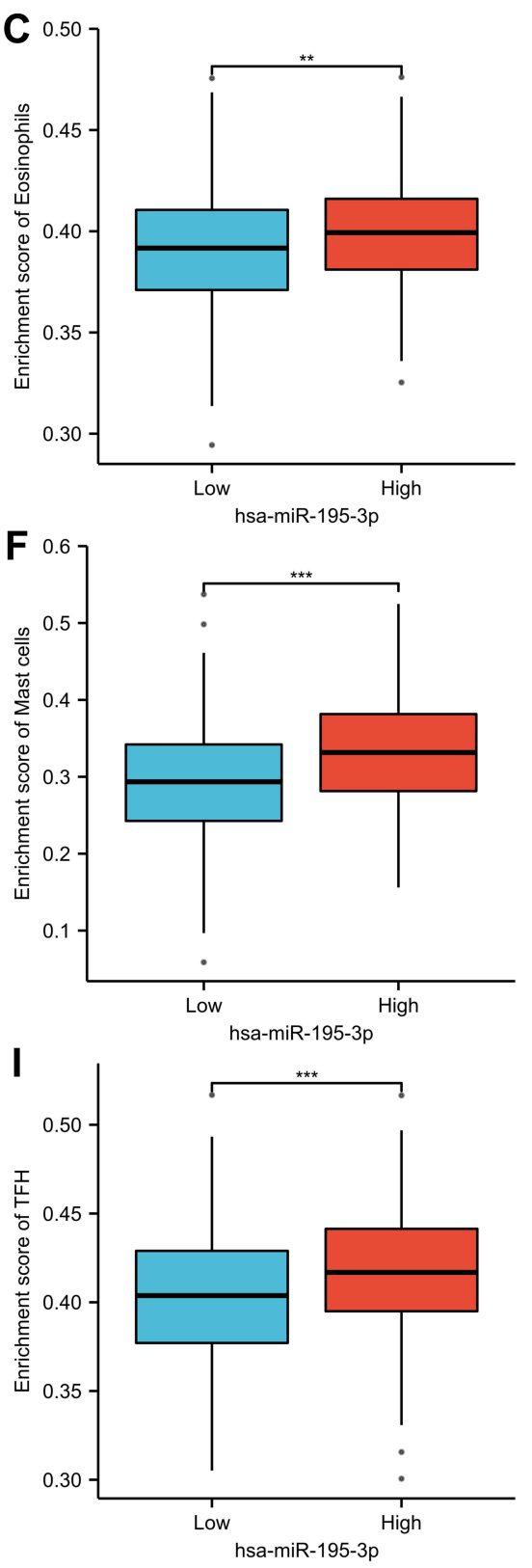

Figure 5 MiR-195-3p expression was associated with 24 LUAD immune cells (grouped comparison plots). (A) B cells, (B) DC, (C) eosinophils, (D) iDC, (E) macrophages, (F) Mast cells, (G) NK cells, (H) pDC, and (I) TFH. ** $\mathrm{P}<0.0$ I; *** $\mathrm{P}<0.00$ I. 

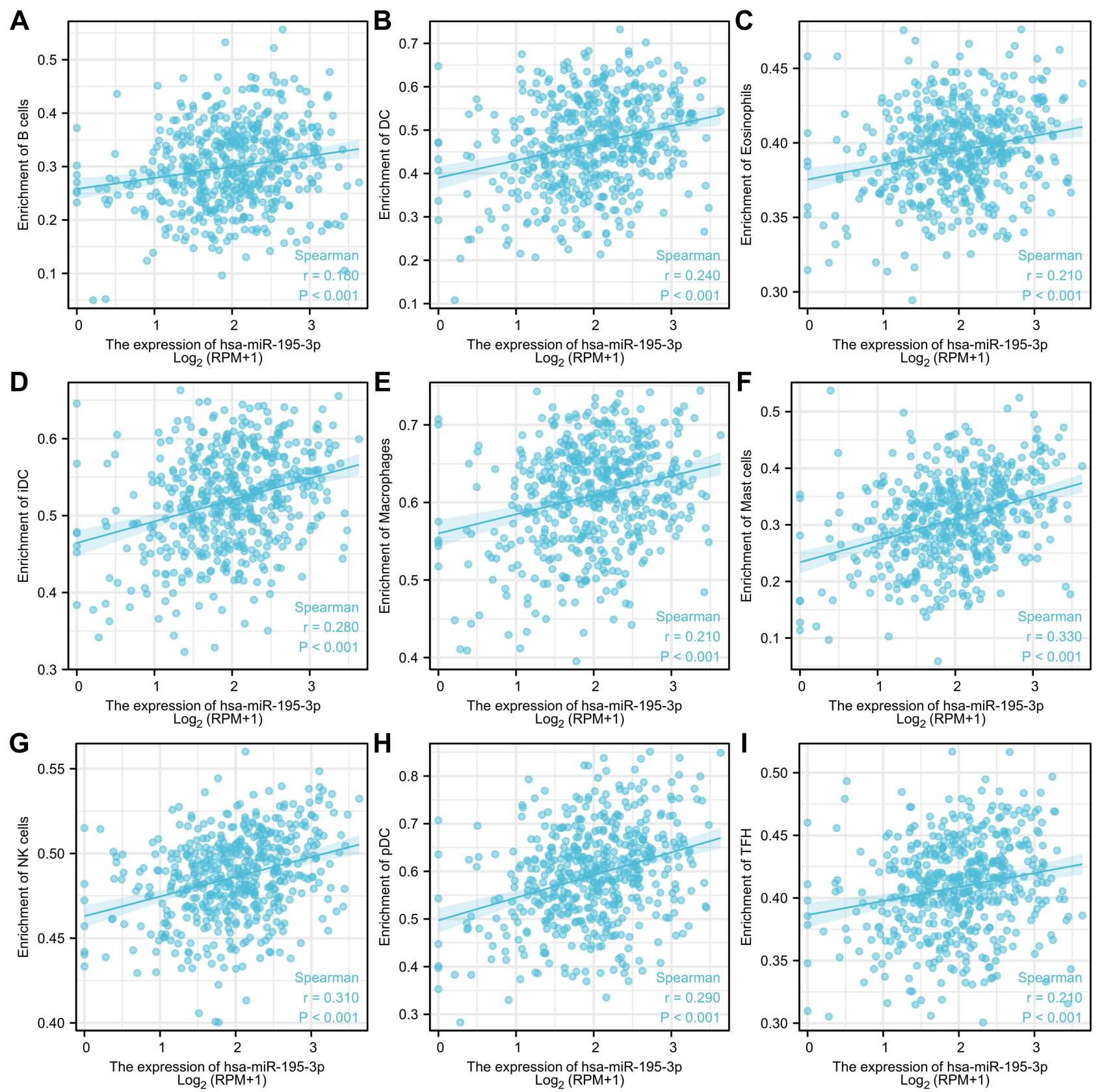

Figure 6 MiR-195-3p expression was associated with 24 LUAD immune cells (scatter plots). (A) B cells, (B) DC, (C) eosinophils, (D) iDC, (E) macrophages, (F) Mast cells, (G) NK cells, (H) pDC, and (I) TFH.

In the present study, miR-195-3p was lowly expressed in LUAD patients, and patients with low miR-195-3p expression had poorer OS. Low miR-195-3p expression was significantly related to $\mathrm{N}$ stage $(\mathrm{P}=0.046)$, pathological stage $(\mathrm{P}=0.011)$, and gender $(\mathrm{P}=0.010)$. The expression of miR-195-3p in LUAD tissue was significantly lower than that in normal lung tissue. The expression of miR-195-3p in patients with advanced $\mathrm{N}$ stage was lower than that in the early stage, and the expression of miR-195-3p in patients with stage III-IV was significantly lower than that of stage I-II. These phenomena suggest that miR-195-3p may be involved in the occurrence of LUAD and inhibit proliferation. Male patients showed low expression of miR-195-3p compared to female patients. The cause of this situation requires further investigation. The low expression of miR-195-3p in LUAD patients is related to poor OS (HR: $0.60 ; 95 \%$ CI: $0.45-0.81 ; \mathrm{P}=0.001$ ) and DSS (HR: $0.55 ; 95 \% \mathrm{CI}$ : 


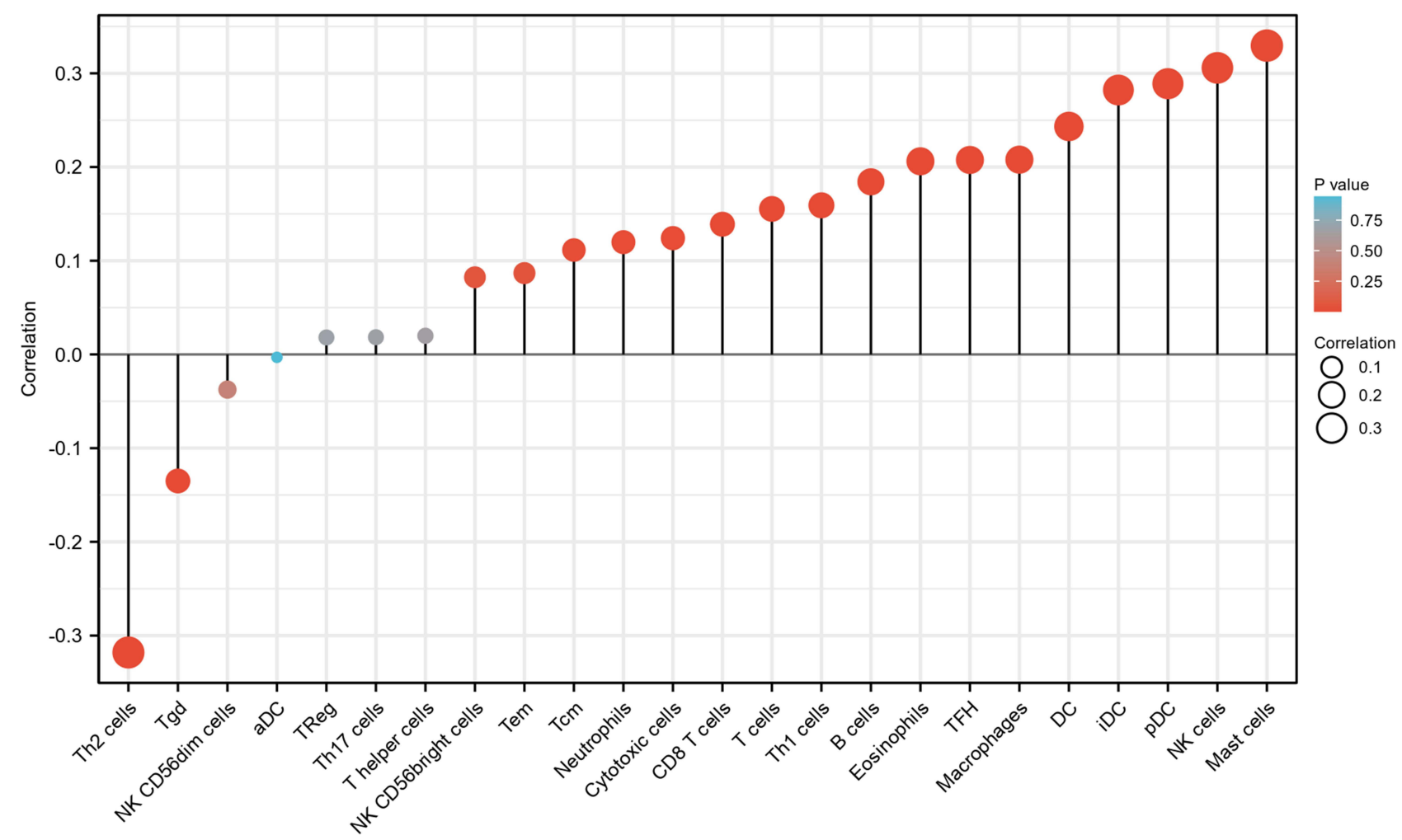

Figure 7 MiR-195-3p expression was associated with 24 LUAD immune cells (Lollipop chart). The point size represents the absolute value of Spearman r.

0.37-0.80; $\mathrm{P}=0.002$ ). And miR-195-3p (HR: 0.488; 95\% CI: $0.304-0.784 ; \mathrm{P}=0.003$ ) were independently correlated with OS in multivariate analysis. High expression of miR195-3p targets, including ABCC2, AGMAT, ARNTL2, ATP6V0A4, CDC25A， CDK1， FAM111B， GJB2, GRIP1, HMGA2, HOXA9, KIF14, SYT2, and TFAP2A, were significantly associated with poor OS in LUAD patients.

MiR-195-3p inhibits cervical cancer cell proliferation by targeting BCDIN3D. ${ }^{31}$ Chemokine CCL4 regulates miR-195-3p to induce VEGF-C expression and lymphangiogenesis in oral squamous cell carcinoma (OSCC). ${ }^{32} \mathrm{In}$ this study, GO and KEGG analysis suggested that miR$195-3 p$ was related to the phagosome pathway. The molecular mechanism of miR-195-3p-mediated development of LUAD needs to be further investigated.

The immunological component of TME acts as a double-edged sword that can inhibit or promote tumor development. ${ }^{33}$ Infiltrating immune cells in TME is what influences tumor growth and progression, as well as treatment outcome and patient prognosis. ${ }^{34}$ The results of this study showed that miR-195-3p was associated with infiltration of B cells, DC, Eosinophils, iDC, Macrophages, Mast cells, NK cells, pDC, and TFH in LUAD. MiR-195-
$3 p$ may promote the function of B cells, DC, Eosinophils, iDC, Macrophages, Mast cells, NK cells, pDC, and TFH.

The present study had the limitation that the current analysis was based on LUAD tissues from the TCGA database. The results of the bioinformatics analysis need to be demonstrated by major bench experiments. In the real world, the clinical and biological significance of miR195-3p in LUAD patients need to be further investigated.

\section{Conclusion}

The expression of miR-195-3p in LUAD tissue was significantly lower than that in normal lung tissue. The low expression of miR-195-3p in LUAD patients is associated with poor OS. MiR-195-3p was involved in the development of LUAD through the phagosome pathway. The expression of miR-195-3p was significantly associated with immune infiltration. The findings of this study suggested that miR-195-3p could be a novel prognostic biomarker for LUAD.

\section{Data Sharing Statement}

The datasets generated and analyzed during the present study are available from the corresponding author on reasonable request. 


\section{Ethics Approval}

This study was submitted to Medical Ethics Committee of Central Hospital of Guangdong Nongken for review and the ethics requirement was waived (Approval NO: [2021]02).

\section{Acknowledgment}

The data set generated in this study can be obtained from TCGA, which provides free resources. The authors thank MyGene Diagnostics Co., Ltd. for technology support.

\section{Author Contributions}

All authors made a significant contribution to the work reported, whether that is in the conception, study design, execution, acquisition of data, analysis and interpretation, or in all these areas; took part in drafting, revising or critically reviewing the article; gave final approval of the version to be published; have agreed on the journal to which the article has been submitted; and agree to be accountable for all aspects of the work.

\section{Funding}

There is no funding to report.

\section{Disclosure}

The authors declare that they have no conflict of interest.

\section{References}

1. Zhang S, Liu J, Yuan T, et al. Circular RNA 0001313 knockdown suppresses non-small cell lung cancer cell proliferation and invasion via the microRNA-452/HMGB3/ERK/MAPK axis. Int $J$ Gen Med. 2020;13:1495-1507. doi:10.2147/IJGM.S272996

2. Bray F, Ferlay J, Soerjomataram I, et al. Global cancer statistics 2018: GLOBOCAN estimates of incidence and mortality worldwide for 36 cancers in 185 countries. CA Cancer J Clin. 2018;68(6):394-424. doi:10.3322/caac. 21492

3. Johnson JL, Pillai S, Chellappan SP. Genetic and biochemical alterations in non-small cell lung cancer. Biochem Res Int. 2012;2012:940405. doi:10.1155/2012/940405

4. Matsuda T, Machii R. Morphological distribution of lung cancer from Cancer Incidence in Five Continents Vol. X. Jpn J Clin Oncol. 2015;45(4):404. doi:10.1093/jjco/hyv041

5. Guo Z, Huang J, Wang Y, et al. Analysis of expression and its clinical significance of the secreted phosphoprotein 1 in lung adenocarcinoma. Front Genet. 2020;11:547. doi:10.3389/fgene.2020.00547

6. Berardi R, Rinaldi S, Santoni M, et al. Prognostic models to predict survival in patients with advanced non-small cell lung cancer treated with first-line chemo- or targeted therapy. Oncotarget. 2016;7 (18):26916-26924. doi:10.18632/oncotarget.8309

7. Franceschini JP, Jamnik S, Santoro IL. Survival in a cohort of patients with lung cancer: the role of age and gender in prognosis. $J$ Bras Pneumol. 2017;43(6):431-436. doi:10.1590/s1806-375620160000 00298

8. Lee YS, Dutta A. MicroRNAs in cancer. Annu Rev Pathol. 2009;4:199-227. doi:10.1146/annurev.pathol.4.110807.092222
9. Hemmatzadeh M, Mohammadi H, Karimi M, et al. Differential role of microRNAs in the pathogenesis and treatment of Esophageal cancer. Biomed Pharmacother. 2016;82:509-519. doi:10.1016/j. biopha.2016.05.009

10. Esquela-Kerscher A, Slack FJ. Oncomirs - microRNAs with a role in cancer. Nat Rev Cancer. 2006;6(4):259-269. doi:10.1038/nrc1840

11. Jin L, Li X, Li Y, et al. Identification of miR-195-3p as an oncogene in RCC. Mol Med Rep. 2017;15(4):1916-1924. doi:10.3892/ mmr.2017.6198

12. Hanahan D, Coussens LM. Accessories to the crime: functions of cells recruited to the tumor microenvironment. Cancer Cell. 2012;21 (3):309-322. doi:10.1016/j.ccr.2012.02.022

13. Bindea G, Mlecnik B, Tosolini M, et al. Spatiotemporal dynamics of intratumoral immune cells reveal the immune landscape in human cancer. Immunity. 2013;39(4):782-795. doi:10.1016/j. immuni.2013.10.003

14. Liu B, Qu J, Xu F, et al. MiR-195 suppresses non-small cell lung cancer by targeting CHEK1. Oncotarget. 2015;6(11):9445-9456. doi:10.18632/oncotarget. 3255

15. Yuan C, Xiang L, Bai R, et al. MiR-195 restrains lung adenocarcinoma by regulating $\mathrm{CD} 4+\mathrm{T}$ cell activation via the $\mathrm{CCDC} 88 \mathrm{C} / \mathrm{Wnt}$ signaling pathway: a study based on The Cancer Genome Atlas (TCGA), Gene Expression Omnibus (GEO) and bioinformatic analysis. Ann Transl Med. 2019;7(12):263.

16. Chen B, Jin X, Wang H, et al. Expression, clinical significance, and prospective pathway signaling of miR-501-3p in ovarian cancer based on database and informatics analysis. Int $J$ Gen Med. 2021;14:5193-5201. doi:10.2147/IJGM.S327673

17. Chen T, Zhu C, Wang X, et al. LncRNA ELF3-AS1 is a prognostic biomarker and correlated with immune infiltrates in hepatocellular carcinoma. Can J Gastroenterol Hepatol. 2021;2021:8323487. doi: $10.1155 / 2021 / 8323487$

18. Liu J, Lichtenberg T, Hoadley KA, et al. An integrated TCGA pan-cancer clinical data resource to drive high-quality survival outcome analytics. Cell. 2018;173(2):400-416.e11. doi:10.1016/j. cell.2018.02.052

19. Agarwal V, Bell GW, Nam J-W, et al. Predicting effective microRNA target sites in mammalian mRNAs. eLife. 2015;4:e05005. doi:10.7554/eLife.05005

20. Chiang HR, Schoenfeld LW, Ruby JG, et al. Mammalian microRNAs: experimental evaluation of novel and previously annotated genes. Genes Dev. 2010;24(10):992-1009. doi:10.1101/ gad. 1884710

21. Liu W, Wang X. Prediction of functional microRNA targets by integrative modeling of microRNA binding and target expression data. Genome Biol. 2019;20(1):18. doi:10.1186/s13059-019-1629-z

22. Krek A, Grün D, Poy MN, et al. Combinatorial microRNA target predictions. Nat Genet. 2005;37(5):495-500. doi:10.1038/ng1536

23. Lu X, Li G, Liu S, et al. Bioinformatics analysis of KIF1A expression and gene regulation network in ovarian carcinoma. Int $J$ Gen Med. 2021;14:3707-3717. doi:10.2147/IJGM.S323591

24. Huang da W, Sherman BT, Lempicki RA. Systematic and integrative analysis of large gene lists using DAVID bioinformatics resources. Nat Protoc. 2009;4(1):44-57. doi:10.1038/nprot.2008.211

25. Huang da W, Sherman BT, Lempicki RA. Bioinformatics enrichment tools: paths toward the comprehensive functional analysis of large gene lists. Nucleic Acids Res. 2009;37(1):1-13. doi:10.1093/nar/ gkn923

26. Hänzelmann S, Castelo R, Guinney J. GSVA: gene set variation analysis for microarray and RNA-seq data. BMC Bioinform. 2013;14:7. doi:10.1186/1471-2105-14-7

27. Gan T-Q,Chen WJ, Qin H, et al. Clinical value and prospective pathway signaling of MicroRNA-375 in lung adenocarcinoma: a study based on the Cancer Genome Atlas (TCGA), Gene Expression Omnibus (GEO) and bioinformatics analysis. Med Sci Monit. 2017;23:2453-2464. 
28. Yong-Hao Y, Xian-guo W, Ming X, et al. Expression and clinical significance of miR-139-5p in non-small cell lung cancer. $J$ Int Med Res. 2019;47(2):867-874. doi:10.1177/0300060518815379

29. Gu W, Fang S, Gao L, et al. Clinic significance of microRNA-99a expression in human lung adenocarcinoma. J Surg Oncol. 2013;108 (4):248-255. doi:10.1002/jso.23381

30. Świtlik WZ, Karbownik MS, Suwalski M, et al. Serum miR-210-3p as a potential noninvasive biomarker of lung adenocarcinoma: a Preliminary Study. Genet Test Mol Biomarkers. 2019;23 (5):353-358. doi:10.1089/gtmb.2018.0275

31. Jin M, Wang L, Zheng T, et al. MiR-195-3p inhibits cell proliferation in cervical cancer by targeting BCDIN3D. J Reprod Immunol. 2021;143:103211. doi:10.1016/j.jri.2020.103211
32. Lien MY, Tsai H-C, Chang A-C, et al. Chemokine CCL4 induces vascular endothelial growth factor c expression and lymphangiogenesis by miR-195-3p in oral squamous cell carcinoma. Front Immunol. 2018;9:412. doi:10.3389/fimmu.2018.00412

33. Angell H, Galon J. From the immune contexture to the Immunoscore: the role of prognostic and predictive immune markers in cancer. Curr Opin Immunol. 2013;25(2):261-267. doi:10.1016/j.coi.2013.03.004

34. Oliver AJ, Lau PKH, Unsworth AS, et al. Tissue-dependent tumor microenvironments and their impact on immunotherapy responses. Front Immunol. 2018;9:70. doi:10.3389/fimmu.2018.00070

\section{Publish your work in this journal}

The International Journal of General Medicine is an international, peer-reviewed open-access journal that focuses on general and internal medicine, pathogenesis, epidemiology, diagnosis, monitoring and treatment protocols. The journal is characterized by the rapid reporting of reviews, original research and clinical studies across all disease areas. The manuscript management system is completely online and includes a very quick and fair peer-review system, which is all easy to use. Visit http://www.dovepress.com/ testimonials.php to read real quotes from published authors.

Submit your manuscript here: https://www.dovepress.com/international-journal-of-general-medicine-journal 